\title{
Chemical Pleurodesis Using a Viscum album Extract in an Infant with Postoperative Chylothorax: A Case Report
}

\author{
Taeyoung Yun, M.D., Hye Won Kwon, M.D., Samina Park, M.D., Woong-Han Kim, M.D., Ph.D. \\ Department of Thoracic and Cardiovascular Surgery, Seoul National University Hospital, Seoul National University College of Medicine, Seoul, Korea
}

\section{ARTICLE INFO}

Received August 12, 2021

Revised October 25, 2021

Accepted November 6, 2021

Corresponding author

Hye Won Kwon

Tel $82-2-2072-4306$

Fax 82-2-764-3664

E-mail 66072@snuh.org

ORCID

https://orcid.org/0000-0002-4949-9688

\begin{abstract}
Chylothorax after thoracic surgery is a rare complication, and treatment for refractory chylothorax is challenging. We report a case of chylothorax after cardiothoracic surgery in an infant after failure of conservative management and thoracic duct ligation. The patient underwent chemical pleurodesis with a Viscum album extract. The treatment was successful and chylothorax did not recur.
\end{abstract}

Keywords: Chylothorax, Pleurodesis, Viscum album, Congenital heart disease, Case report

\section{Case report}

A 17-day-old girl (body weight, $1.99 \mathrm{~kg}$ ) previously diagnosed with Edwards syndrome, esophageal atresia, and congenital heart disease (coarctation of aorta, patent ductus arteriosus, large perimembranous ventricular septal defect, and atrial septal defect), underwent 1-stage total repair of congenital heart disease via sternotomy. She had undergone transesophageal fistula ligation and esophagus anastomosis via right thoracotomy on the first day of life.
With the patient experiencing aggravated heart failure, her parents requested aggressive treatment. We decided to perform cardiac surgery in a 1-stage total repair.

On postoperative day 3 after cardiac surgery, a chest tube was newly inserted to drain right pleural effusion, which was confirmed by chest radiography (Fig. 1A). The patient was extubated on postoperative day 7 and started feeding the next day. Two days later, when full feeding was reached, the color of the chest tube drainage became milky and chylothorax was confirmed by a high triglyceride level $(358 \mathrm{mg} / \mathrm{dL})$.
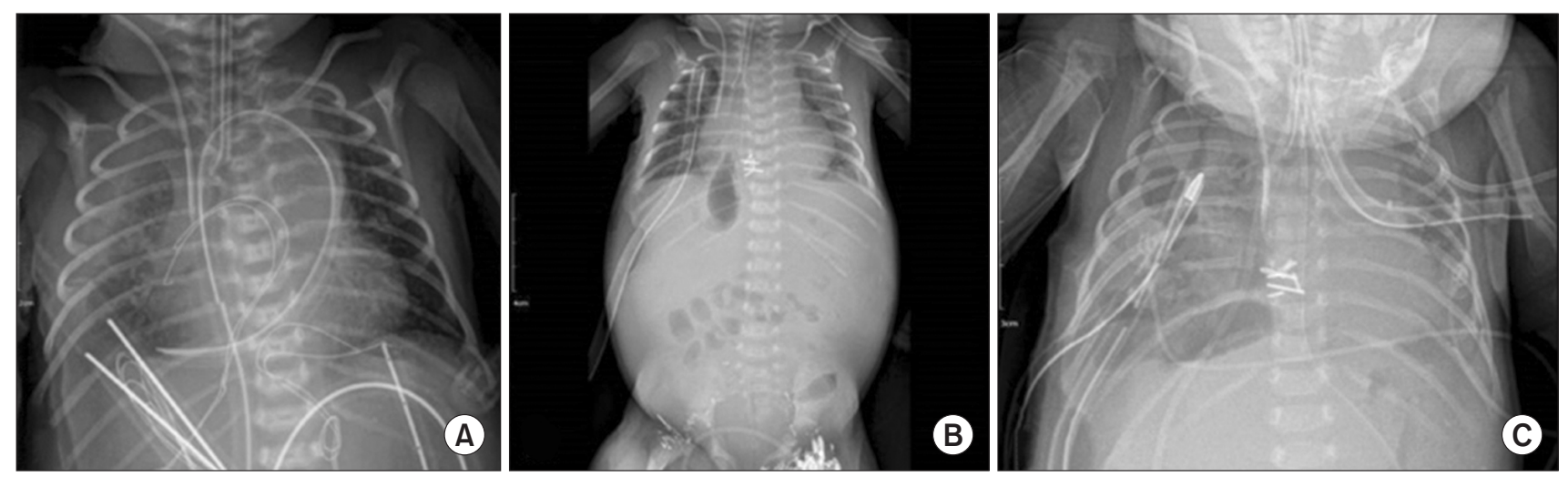

Fig. 1. (A) Increased right pleural effusion on postoperative day 3 after cardiac surgery. (B) Abdominal distension with suspicious chylous ascites on postoperative day 1 after the second thoracic duct ligation. (C) Newly inserted chest tube for loculated right pleural effusion at 1 day after pleurodesis. 
Conservative treatment was initiated with a medium-chain triglyceride (MCT) formula. Nonetheless, the fluid drainage remained 60 to $90 \mathrm{~mL}$ per day (30-45 mL/kg per day). We decided to start total enteric rest accompanied by continuous intravenous octreotide infusion, with a starting dose of $4 \mu \mathrm{g} / \mathrm{kg}$ per hour to a maximum dose of $6 \mu \mathrm{g} / \mathrm{kg}$ per hour, but it was not effective. Due to refractory chylothorax, we performed thoracic duct ligation surgery via right thoracotomy, but the procedure was not successful, and the pleural drainage increased to $242 \mathrm{~mL}$ per day. We also attempted thoracic duct lymphangiography for embolization. However, due to the small peripheral thoracic duct, the punctured lymph node ruptured during the injection of lipiodol (oil contrast agent), and lymphangiography was not possible. An attempt was made to puncture the upper-level lymph node using the transabdominal approach, but it failed. In order to perform lipiodol injection by precisely localizing the leakage site through direct thoracic access during surgery, we decided to perform a secondary thoracic duct ligation. Injections could not be made during surgery because the site of the leakage was unclear. However, duct ligation was performed with an endoclip at the site of suspected chyle leakage and an adhesive surgical fibrin sealant (Tisseel; Baxter, Westlake Village, CA, USA) was applied. The drainage decreased to $100 \mathrm{~mL}$ per day after the second surgery, but chylous ascites developed. With ongoing respiratory acidosis even at the maximal ventilator setting with ascites drainage, a high-frequency ventilator was applied and prolonged ventilator care became necessary (Fig. 1B). On postoperative day 5, after the second thoracic ligation surgery, octreotide infusion was restarted. However, on postoperative day 13, pleural drainage increased to $200 \mathrm{~mL}$ per day with no improvement of chylous ascites after intermittent paracentesis. Since there was no response to prolonged total enteral rest and octreotide infusion treatment, we decided to perform chemical pleurodesis with $5 \mathrm{mg}$ of Viscum album, mistletoe extraction (Abnova Viscum Fraxini Injection; ABNOBA GmbH, NiefernOschelbronn, Germany) on postoperative day 6. The amount of pleural drainage steadily decreased from 100 $\mathrm{mL}$ per day to $20 \mathrm{~mL}$ per day (Fig. 2). On the first day after Viscum album pleurodesis, a loculated pleural fluid collection was observed on chest radiography and ultrasonography (Fig. 1C); therefore, we inserted an additional chest tube for drainage. Over the next 7 days, the drainage decreased significantly to $10 \mathrm{~mL}$ per day for each chest tube, so the newly inserted tube was removed first. After 7 days after the first tube removal, the last remaining tube was removed with no additional drainage. For prolonged ventilator care, tracheostomy was performed 1 month later. The patient was weaned from mechanical ventilation, 9 days after tracheostomy and was discharged home with no recurrence of chylothorax or secondary radiologic changes (Fig. 3).

The case was approved by the Institutional Review Board of Seoul National University Hospital as a minimal-risk retrospective study (approval no., H-2107-129-1236) that did not require individual consent based on the institutional guidelines for waiving consent.

\section{Discussion}

After congenital heart surgery, it has been reported that $2.8 \%$ to $9.2 \%$ of patients suffer from chylothorax $[1,2]$. Prolonged chylothorax is associated with poor nutrition, electrolyte imbalances, an increased length of hospital stay,

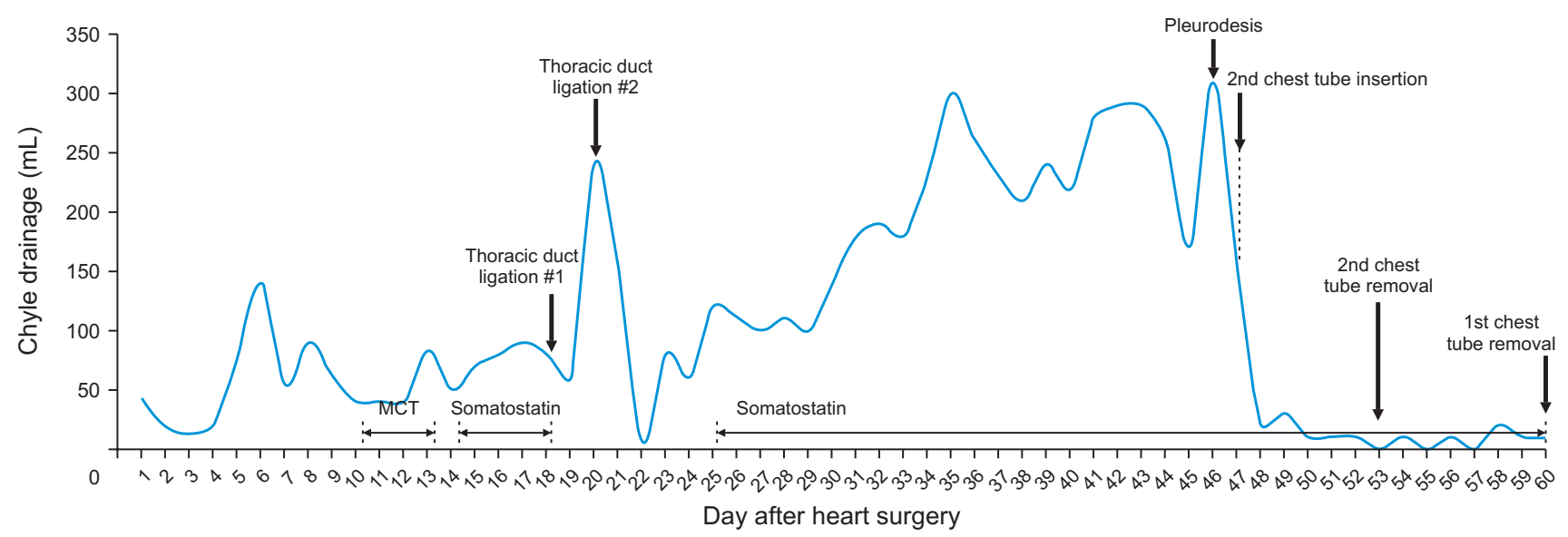

Fig. 2. Daily chyle drainage, from the day of cardiac surgery to the day of chest tube removal. MCT, medium-chain triglyceride. 

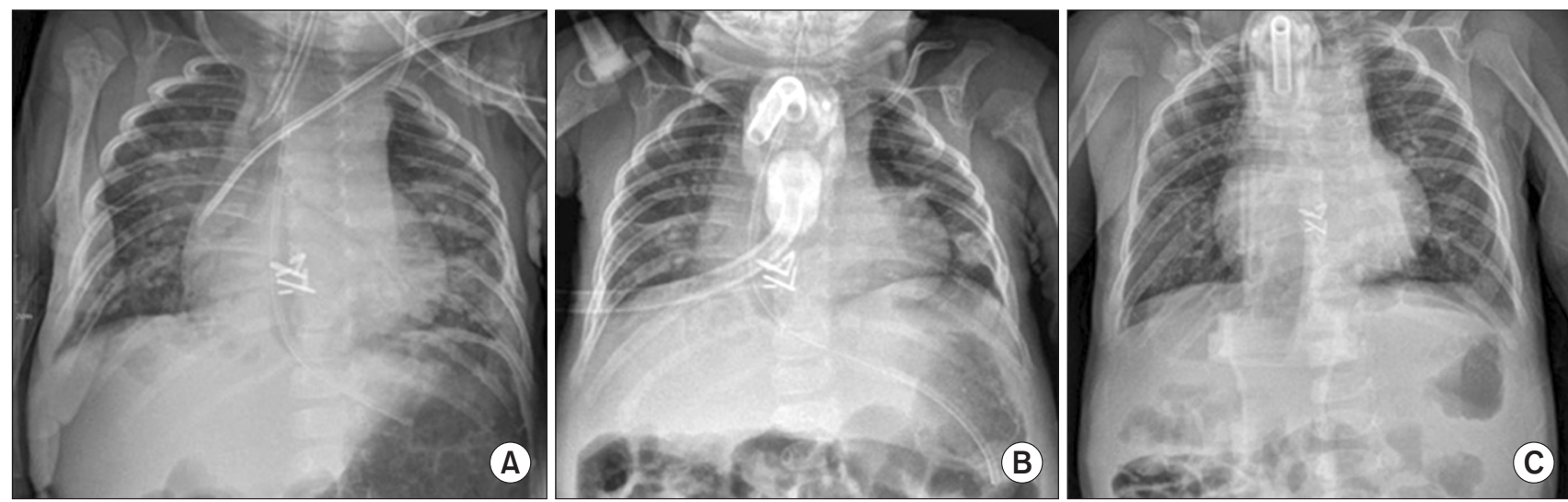

Fig. 3. (A) All chest tubes were removed at 13 days after pleurodesis. (B) Chest radiograph at discharge at 2 months after pleurodesis. (C) Chest radiograph at outpatient follow-up (patient age, 13 months).

prolonged mechanical ventilation, and a high risk of mortality [1-3]. With no standardized treatment guidelines, traditional treatments include pleural drainage, an MCT or low-fat diet, and enteric rest with total parenteral nutrition. Other treatment options include infusion of octreotide to decrease splanchnic blood flow to reduce the triglyceride content of chyle, which was reported to be successful in $29 \%$ to $62 \%$ of pediatric patients $[4,5]$. For refractory chylothorax, thoracic duct ligation is considered after failure of medical treatment [1-3]. Lymphangiography and thoracic duct embolization have recently been considered as less invasive alternatives to surgery [6]; however, there are technical difficulties in small infants weighing less than $3 \mathrm{~kg}$, as in this case. Therefore, chemical pleurodesis can be a treatment option for refractory chylothorax of infants who do not respond to conventional and surgical treatment. The infusion of a chemical agent through the inserted chest tube creates pleural adhesions that obliterate the leakage of chyle. Agents used for pleurodesis include antibiotics, antiseptics (povidone-iodine), talc, and fibrin glue [7]. However, there is no efficient sclerosant that shows strong pro-adhesive effects but no pro-inflammatory activity [7]. Furthermore, there is no standard agent recommended for neonates and infants that has few side effects.

Viscum album is an extract of European mistletoe, a semiparasitic plant that grows on host trees of the genus Fraxinus [8]. It has been used as an anticancer adjuvant treatment for about 80 years in European countries, and it contains a variety of biologically active compounds that display cytotoxic effects and stimulate the immune system [8]. It also has been used for chemical pleurodesis in patients with malignant pleural effusion, in whom it was reported to show favorable results [9]. A case report de- scribed intrapleural instillation of a Viscum album extract in infants with congenital chylothorax that was refractory to conventional management [10].

In this case, we started standard conservative treatment with pleural fluid drainage and enteral feeding with an MCT formula. Next, an octreotide intravenous infusion was started with enteric rest and total parenteral nutrition. In addition, we performed surgical ligation of the thoracic duct twice. Nevertheless, none of these treatments were successful. By using Viscum album for pleurodesis, in this case, successful treatment was achieved by a mild inflammatory reaction that was sufficient to induce adequate adhesions in the of pleural cavity. Since Viscum album is an extract from mistletoe with an anticancer effect, it is considered to have low toxicity. This case implies that using Viscum album for pleurodesis can be a treatment choice for refractory postoperative chylothorax in an infant. There were no side effects or recurrence during follow-up until the age of 13 months. Chyle drainage decreased dramatically, and all the chest tubes were removed within 2 weeks after pleurodesis. In conclusion, chemical pleurodesis using Viscum album extract could be a safe and effective treatment option for infants with postoperative chylothorax refractory to conventional management.

\section{Conflict of interest}

No potential conflict of interest relevant to this article was reported.

\section{ORCID}

Taeyoung Yun: https://orcid.org/0000-0002-4303-8493 
Hye Won Kwon: https://orcid.org/0000-0002-4949-9688

Samina Park: https://orcid.org/0000-0001-9625-2672

Woong-Han Kim: https://orcid.org/0000-0003-2837-7929

\section{References}

1. Mery CM, Moffett BS, Khan MS, et al. Incidence and treatment of chylothorax after cardiac surgery in children: analysis of a large multi-institution database. J Thorac Cardiovasc Surg 2014;147:67886.

2. Biewer ES, Zurn C, Arnold R, et al. Chylothorax after surgery on congenital heart disease in newborns and infants: risk factors and efficacy of MCT-diet. J Cardiothorac Surg 2010;5:127.

3. Costa KM, Saxena AK. Surgical chylothorax in neonates: management and outcomes. World J Pediatr 2018;14:110-5.

4. Aljazairi AS, Bhuiyan TA, Alwadai AH, Almehizia RA. Octreotide use in post-cardiac surgery chylothorax: a 12-year perspective. Asian Cardiovasc Thorac Ann 2017;25:6-12.
5. Rosti L, De Battisti F, Butera G, et al. Octreotide in the management of postoperative chylothorax. Pediatr Cardiol 2005;26:440-3.

6. Schild HH, Naehle CP, Wilhelm KE, et al. Lymphatic interventions for treatment of chylothorax. Rofo 2015;187:584-8.

7. Mierzejewski M, Korczynski P, Krenke R, Janssen JP. Chemical pleurodesis: a review of mechanisms involved in pleural space obliteration. Respir Res 2019;20:247.

8. Kienle GS, Kiene H. Review article: influence of Viscum album $L$ (European mistletoe) extracts on quality of life in cancer patients: a systematic review of controlled clinical studies. Integr Cancer Ther 2010;9:142-57.

9. Lee YG, Jung I, Koo DH, et al. Efficacy and safety of Viscum album extract (Helixor-M) to treat malignant pleural effusion in patients with lung cancer. Support Care Cancer 2019;27:1945-9.

10. Cho HJ, Na KJ, Kim DW, Choi YE, Ma JS, Jeong IS. Chemical pleurodesis using a Viscum album extract in infants with congenital chylothorax. Eur J Pediatr 2014;173:823-6. 